\title{
Consideraciones en el manejo de la vía aérea en embarazadas
}

\author{
Airway management considerations for pregnant women
}

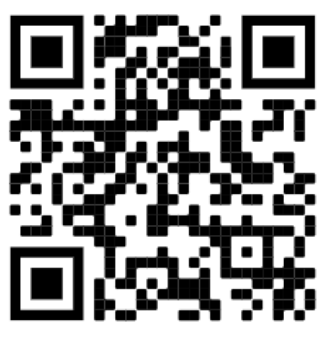

Recibido

$31 / 12 / 2020$

${ }^{1}$ Dra. Mónica Alvarado Ferllini

Investigadora independiente, San José, Costa Rica

(D) https://orcid.org/0000-0002-0975-5157

${ }^{2}$ Dra. Jazmín Montero Fonseca Investigadora independiente, Alajuela, Costa Rica

(iD) https://orcid.org/0000-0003-1319-3449

${ }^{3} \mathrm{Dr}$. Juan Carlos Herrera Pérez Investigador independiente, Alajuela, Costa Rica

(i) https://orcid.org/0000-0002-5426-9107

Corregido $10 / 01 / 2021$

Aceptado $15 / 01 / 2021$

\section{RESUMEN}

La anatomía y fisiología de las mujeres embarazadas sufre modificaciones en la mayoría de sistemas del cuerpo, pero algunos de los cambios más significativos son los que ocurren a nivel respiratorio, los cuales convierten a las pacientes obstétricas en una potencial vía aérea difícil. Esto debe ser identificado mediante una evaluación completa de la vía aérea previo a la manipulación de esta, al igual que como se realiza en pacientes no gestantes. Posterior a dicha valoración, es preciso aplicar los algoritmos que han sido diseñados para orientar el manejo de la ventilación e intubación en este tipo de pacientes, por ejemplo, las guías publicadas por la Asociación de Anestesiólogos Obstétricos y la Sociedad de Vía Aérea Difícil, siempre con el objetivo de preservar al máximo el bienestar materno y fetal.

PALABRAS CLAVE: manejo de la vía aérea; mujeres embarazadas; anestesia obstétrica; ventilación pulmonar; intubación.

\section{ABSTRACT}

The anatomy and physiology of pregnant women undergoes changes in most systems of the body, but some of the most significant changes are those that occur at the respiratory level, which make obstetric patients a potential difficult airway. This should be identified by a complete airway evaluation prior to manipulation, as it is done in non-pregnant patients. After this assessment, it is necessary to apply the algorithms that have been designed to orientate the management of ventilation and intubation in this type of patient, for example, the guidelines published by Obstetric Anaesthetists' Association and Difficult Airway Society, always with the intention of preserving the maximum maternal and fetal welfare.

\footnotetext{
${ }^{1}$ Médica general, graduada de la Universidad de Costa Rica (UCR), cod. MED16825. Correo: monicaferllini@hotmail.com

${ }^{2}$ Médica general, graduada de la Universidad de Costa Rica (UCR), cod. MED16818. Correo: jazmf15@gmail.com

${ }^{3}$ Médico general, graduado de la Universidad de Costa Rica (UCR). cod. MED16809. Correo: juan.herreraperez@ucr.ac.cr
} 
KEYWORDS: airway management; pregnant women; anesthesia, obstetrical; pulmonary ventilation; intubation.

\section{INTRODUCCIÓN}

El embarazo se asocia con cambios significativos en la anatomía y fisiología de la mujer, los cuales afectan la habilidad de la paciente de mantener una adecuada oxigenación en tiempos de compromiso respiratorio, además, afecta la capacidad de los médicos de asegurar adecuada ventilación y oxigenación cuando la intubación endotraqueal es necesaria (1), por lo tanto, el manejo de las pacientes embarazadas difiere de otros pacientes en muchos aspectos y esto implica que un evento potencialmente letal podría ocurrir durante 9 meses (2). La tasa de intubación traqueal fallida en obstetricia se ha mantenido sin cambios en las últimas cuatro décadas (3): se reporta que la incidencia de la vía aérea difícil y/o fallida en la paciente embarazada en promedio está entre 1:250 a 1:300, es decir, es 10 veces superior que en la población general (4). Debido a lo anterior, el manejo de la vía aérea en la paciente embarazada sigue siendo un reto, no obstante, la elaboración de algoritmos, agrupaciones de estudio en la materia y el desarrollo de nuevos dispositivos avanzados han sido de ayuda para la ventilación e intubación endotraqueal (4). Con esta finalidad, la primera guía para manejo de intubación fallida en obstetricia fue publicada en 1976, desde entonces, se han realizado diferentes modificaciones a las guías, incluyendo las publicadas en 2015 por la Asociación de Anestesiólogos Obstétricos y la Sociedad de Vía Aérea Difícil (OAA-DAS), que continúan vigentes (3). Con base en lo antes mencionado, el objetivo de la presente revisión es describir las principales variantes en la anatomía y fisiología de las pacientes embarazadas, además de la correcta evaluación y manejo de la vía aérea en este tipo de pacientes, que eviten fallos 0 situaciones que puedan comprometer la vida de la madre y el producto.

\section{MÉTODO}

Para la elaboración de este artículo se llevó a cabo una revisión bibliográfica con artículos provenientes principalmente de las bases de datos PubMed, Elsevier, Springer, Wiley Online Library, SciELO y Medigraphic, así como tres libros médicos y una página web. Los documentos consultados se encuentran en español o inglés, todos ellos publicados entre noviembre 2015 y diciembre 2020.

En la búsqueda se utilizaron los términos "Vía aérea en embarazadas", "Vía aérea difícil", combinados con "obstetricia", "manejo", "fisiología", "anatomía" "evaluación”, "ventilación”, "intubación”. La revisión se centró en la información más actualizada y pertinente para exponer los temas tratados en este artículo.

\section{CAMBIOS ANATÓMICOS Y FISIOLÓGICOS QUE INFLUYEN EN LA VÍA AÉREA DURANTE EL EMBARAZO}

El embarazo produce alteraciones importantes en casi cada sistema del cuerpo, pero algunos de los cambios más notorios ocurren a nivel respiratorio, los cuales pueden afectar el manejo de la vía aérea ( 5 , 6): 
1. Cavidad nasal: ocurre edema y friabilidad en nasofaringe y orofaringe, secundario a ingurgitación capilar, la cual inicia en el primer trimestre y aumenta progresivamente durante el embarazo. El efecto de los estrógenos en la mucosa nasal predispone a trauma, sangrado y obstrucción $(1,5)$.

2. Laringe: durante el primer y segundo trimestre, la voz es redondeada y con buena vibración, pero durante el tercer trimestre la fatiga de las cuerdas vocales es más prevalente, lo que causa una disminución en el máximo tiempo de fonación (1). También, se restringe el tamaño de la apertura glótica (5).

3. Faringe: ocurre edema faríngeo, debido al aumento del agua corporal total y retención de fluidos por efecto de la progesterona $(1,6)$. Asimismo, este fenómeno puede acentuarse de manera aguda durante la labor de parto, ya que aumenta el volumen de tejidos blandos rodeando la vía aérea, incluida la tráquea (1). Como consecuencia, el grado de Mallampati se modifica durante la gestación y puede aumentar hasta grado IV entre las 12 y 38 semanas (4).

4. Caja torácica: debido al efecto de la relaxina, se presenta laxitud de los ligamentos, incluyendo los de las costillas inferiores $(5,1)$. Por lo tanto, los diámetros anteroposterior y transverso de la pared torácica aumentan durante el embarazo cerca de $2 \mathrm{~cm}$ y el resultante incremento de 5-7 cm en la circunferencia de la caja torácica a nivel de las costillas inferiores crea una posición casi horizontal. De igual manera, aumenta al ángulo subcostal un $55 \%$ y el incremento de la presión intraabodominal desplaza el diafragma hacia arriba aproximadamente $4 \mathrm{~cm}$, lo cual acrecienta el volumen intratorácico (1).

5. Función pulmonar estática: como el desplazamiento del diafragma es compensado por el aumento en las dimensiones lateral y anteroposterior del tórax, la capacidad pulmonar total (CPT) permanece sin cambios o disminuye mínimamente. Sin embargo, sí ocurren algunos cambios en los volúmenes y capacidades pulmonares: la capacidad residual funcional (CRF) se reduce un $20-30 \%$, el volumen residual (VR) disminuye $20-25 \%$ y el volumen corriente (VC) aumenta 30-50\% (1,7). A la capacidad inspiratoria se le suma entre 5 y $10 \%$ durante el segundo y tercer trimestre, mientras que la frecuencia respiratoria se mantiene mayoritariamente sin cambios (1).

6. Ventilación e intercambio de gases: la ventilación minuto aumenta 40-50\%, como consecuencia de la progesterona, también, aumenta la quimiosensibilidad al dióxido de carbono (CO2) debido al aumento del impulso respiratorio (respiratory drive) (8, 1). La hiperventilación en el embarazo es mediada por progesterona y ocurre en respuesta al aumento de tasa metabólica y producción de $\mathrm{CO} 2$. Por lo tanto, la disnea es más común en el tercer trimestre, debido a este aumento del impulso respiratorio (1).

7. Consumo de oxígeno (VO2) y producción de dióxido de carbono (VCO2): por un lado, el VO2 aumenta 20$40 \%$ por incremento de demandas metabólicas por el feto, placenta y útero, 
además, el creciente peso materno amplifica el VO2 en reposo $(1,5)$. Este aumento del VO2, junto con la disminución de la CRF, predisponen a desaturación más rápida y menor tolerancia a la apnea (9). Por otro lado, la VCO2 aumenta 35\%, sin embargo, dado que el incremento de la ventilación minuto excede el de VO2 y VCO2, las presiones parciales alveolares y arteriales de oxígeno (PAO2 y $\mathrm{PaO} 2$ ) aumentan $10 \%$, mientras que las de $\mathrm{CO} 2$ (PACO2 y PaCO2) disminuyen cerca de $30 \mathrm{mmHg}(1,5,10)$. Durante la labor de parto, el dolor lleva a mayor hiperventilación, lo que lleva a mayor disminución de PaCO2 (1).

8. Parámetros ácidos base: la hiperventilación con alcalosis respiratoria lleva a compensación renal con mayor excreción de bicarbonato $(1,8)$, por lo que en el tercer trimestre el bicarbonato llega a ser $12-22 \mathrm{mEq} / \mathrm{L}$. El $\mathrm{pH}$ se mantiene entre 7.42 y 7.46 , es decir, es ligeramente superior. La alcalosis crónica aumenta el 2,3-difosfoglicerato (2,3-DPG), lo que desplaza la curva de disociación de la oxihemoglobina hacia la derecha y la P50 aumenta de 27 a 30 $\mathrm{mmHg}$ : todo esto facilita la entrega de $\mathrm{O} 2$ transplacentario hacia el feto $(1,10)$.

9. Otros cambios: aumentan el peso corporal y el volumen de las mamas, lo cual dificulta la ventilación con máscara, el posicionamiento y la inserción del laringoscopio. Adicionalmente, la relajación del músculo liso gastrointestinal inducida por progesterona, el cambio de posición del estómago, el retraso del vaciamiento gástrico y el tono disminuido del esfínter esofágico inferior agravan el riesgo de broncoaspiración $(3,6)$.

\section{EVALUACIÓN DE LA VÍA AÉREA}

Es importante realizar una evaluación rutinaria de la vía aérea de la embarazada (4). La evaluación orientada a predecir la posibilidad de ventilar y oxigenar es más relevante que la intubación; si bien no existe literatura suficiente que defina predictores de "ventilabilidad" en pacientes embarazadas, se podrían extrapolar de otros grupos, puesto que los factores son similares en pacientes no grávidas $(2,4)$.

Algunos predictores de imposibilidad para ventilación con máscara facial son: obesidad (índice de masa corporal mayor de 30 $\mathrm{kg} / \mathrm{m}^{2}$ ), Mallampati III (solo son visibles el paladar duro y blando) o IV (solamente se observa el paladar duro), limitación severa para la protrusión mandibular, roncar, ser edéntula, preeclampsia, entre otros $(2,4,10)$. En cuanto a la evaluación para predecir la facilidad de la laringoscopía e intubación, algunas variables que condicionan a que sea un procedimiento complejo son las siguientes: cuello corto, cuello grueso (más de 17 pulgadas de circunferencia), incisivos superiores prominentes, micrognatia, Mallampati III o IV, distancia tiromentoniana disminuida (menos de 3 traveses de dedo), dificultad para la flexoextensión del cuello, paladar muy alto o muy bajo, entre otros $(2,4,10,11)$.

\section{MANEJO DE LA VÍA AÉREA EN OBSTETRICIA}

Cada paciente obstétrica debe ser considerada una vía aérea difícil, por lo que es mandatorio tomar precauciones. 
Se debe recordar que la CRF reducida y el aumento de VO2 producen rápida desaturación durante apnea, la visualización de estructuras se puede ver alterada por el edema de mucosas y el aumento de la friabilidad puede ocasionar sangrado. De igual modo, las mamas prominentes pueden contribuir a una difícil intubación $(6,12)$. Por lo tanto, algunas recomendaciones generales son $(6,10,12,13)$ :

- Disponer de un tubo endotraqueal de menor tamaño: se recomiendan tubos de 6 a $6.5 \mathrm{~mm}$.

- Evitar la ruta nasal para prevenir epistaxis.

- La manipulación de la vía aérea debe ser mínima para evitar sangrado y trauma.

- Proveer adecuada preoxigenación sin sobreventilar: es necesario evitar la alcalosis respiratoria.

- La técnica estándar para preoxigenar es con respiraciones con $100 \%$ oxígeno por 3-5 minutos de un volumen corriente, sin embargo, 8 respiraciones profundas en 60 segundos proveen adecuada desnitrogenación.

- El estómago debe ser considerado lleno y se deben tomar precauciones para prevenir aspiración, por ejemplo, deben recibir un antagonista de receptor $\mathrm{H} 2$ la noche previa y en la mañana de la cirugía $(12,1)$. Si requieren anestesia general, el citrato de sodio debe ser administrado antes de la inducción.

- Equipo adicional debe estar disponible, como: videolaringoscopio, tubos endotraqueales más pequeños, dispositivos supraglóticos, entre otros.
- La secuencia de intubación rápida es el estándar debido al riesgo de aspiración.

- En el caso de pacientes obesas o mamomegalia, se recomienda una posición semielevada o "en rampa" colocando sábanas u otros implementos bajo los hombros, cuello y occipucio, con el fin de lograr alinear horizontalmente el meato auditivo externo con la escotadura esternal.

\section{A. Vía aérea difícil sospechada}

Aunque en la gran mayoría de los centros hospitalarios utilizan anestesia regional para las cesáreas, esto no descarta la necesidad eventual de usar anestesia general por anestesia fallida $u$ otra razón (4). En el caso de las pacientes obstétricas, la intubación despierta es la opción más segura, si se espera una vía aérea difícil, particularmente, si también se anticipa ventilación con máscara dificultosa (2). De hecho, las guías de la Sociedad de Vía Aérea Difícil (DAS) para intubación despierto en adultos recomiendan que la intubación traqueal despierto sea considerada en la presencia de predictores de vía aérea difícil, aunque no se trate de embarazadas (14).

Asimismo, en aquellos casos en los cuales se sospeche vía aérea difícil, se deben considerar métodos avanzados: el fibrobroncoscopio óptico flexible (FOB) por vía oral (para evitar epistaxis) es el método estándar en este tipo de pacientes. Sin embargo, puede emplearse videolaringoscopio para intubación despierta con adecuada anestesia tópica 0 los dispositivos supraglóticos (SGA) para facilitar el manejo de la vía aérea con la paciente 
despierta, aunque la calidad de los datos sobre el uso de SGA para cesáreas es baja $(4,15)$. Como ya se mencionó, en el 2015, la OAA y DAS publicaron las guías para el manejo de intubación traqueal difícil y fallida en obstetricia. Ellos desarrollaron 4 algoritmos: el primero enfatiza la importancia de la planificación y preparación y describe las mejores prácticas para la secuencia de intubación rápida y la laringoscopía $(3,13)$. Este primer algoritmo abarca las siguientes operaciones:

- Paso 1: planificación y preparación. Incluye evaluación de la vía aérea, estimación del periodo de ayuno, profilaxis con antiácidos, resucitación fetal intrauterina en los casos que sea preciso, identificar posible ayuda, preparar el equipo para intubación difícil o fallida, entre otros $(3,9)$.

- Paso 2 (secuencia de intubación rápida): se debe situar la paciente en posición de Fowler/rampa y en decúbito lateral izquierdo, preoxigenar con $\mathrm{O} 2$ $100 \%$ o considerar oxigenación nasal, realizar presión cricoidea (10 N, máximo $30 \mathrm{~N}$ ), efectuar una inducción anestésica adecuada y considerar ventilación con máscara con una presión máxima $\left(P_{\text {máx }}\right)$ de $20 \mathrm{cmH} 2 \mathrm{O}(3,9)$.

- Paso 3 (primer intento): en caso de inadecuada visualización laríngea, se debe optimizar reduciendo la presión cricoidea, realizando manipulación laríngea externa, reposicionando la cabeza y cuello o usando un conductor o bougie $(3,9)$.

- Paso 4 (segundo intento): si no se logró la intubación, se debe ventilar con máscara nuevamente, solicitar ayuda, considerar otro tipo de laringoscopio y remover presión cricoidea $(3,9)$.

- Paso 5 (tercer intento): este último intento debe ser realizado de manera exclusiva por un experto $(9,3)$. Debido al riesgo aumentado de sangrado y edematización de la vía aérea, se recomienda limitar los intentos de intubación de 2 a 3 únicamente, en lugar de 3 a 4, como ocurre en la población no obstétrica (13).

- Paso 6: si fallan todas las acciones previas, se debe declarar una intubación traqueal obstétrica fallida y proseguir con el segundo algoritmo de las guías $(3,9,13)$.

B. Vía aérea difícil no sospechada

La forma de proceder en este tipo de situaciones puede basarse en el esquema de manejo de la intubación traqueal fallida, sin embargo, es esencial recordar que es posible saltarse pasos de acuerdo con el bienestar fetal y/o materno. El algoritmo se describe a continuación $(3,16)$ :

- Paso 1: cuando el primer intento de intubación falla, se debe pedir ayuda al profesional más experimentado y enfocarse en reoxigenar. Se debe realizar una ventilación bolsa-máscara con la válvula de limitación de presión ajustable (APL) establecida a $\leq 20$ $\mathrm{cmH} 2 \mathrm{O}$, preferiblemente, con la técnica de dos manos con dos personas $(3,16)$. Si la ventilación está siendo efectiva, se debe intentar una segunda laringoscopía, después de mejorar la posición y liberar la presión cricoidea en una forma gradual (16). El segundo intento debe llevarse a cabo con equipo 
alternativo, por ejemplo, empleando un videolaringoscopio, cambiando las hojas del laringoscopio o con una guía, según la disponibilidad de equipo. Si el segundo intento fracasa, se continúa con el paso $2(3,16)$.

- Paso 2: es necesario enforcarse en mantener la oxigenación a través de una máscara facial o SAD, de preferencia de segunda generación, además de prevenir aspiración y el despertar de la paciente. En el caso de los SAD, deben permitirse un máximo de 2 intentos para colocarlos (16, 3). Una vez posicionados, se procede según la condición materna y fetal: si existe sufrimiento fetal y se trata de una cesárea, se procede a realizar la cirugía con el dispositivo supraglótico, a pesar del riesgo de aspiración. Si la condición de la madre y el producto es estable, se despierta a la madre y se puede hacer uso de bloqueos neuroaxiales o intubación fibróptica. Si la colocación del SAD falla en 2 ocasiones, se procede al paso 3 (16).

- Paso 3: se debe continuar con ventilación con máscara facial utilizando una técnica adecuada y reduciendo la presión cricoidea $(3,16)$. Si no se logra una ventilación efectiva, se trata de un fallo completo de la ventilación y se debe buscar ayuda experta.

- Paso 4: se debe solicitar apoyo adicional y posicionar a la paciente para cricotiroidotomía de emergencia.

C. No ventilación-no intubación

El fallo persistente para intubar y ventilar, a pesar de intentos óptimos con SAD y/o bolsa-máscara, debe ser declarado una emergencia y se debe solicitar ayuda de especialistas como otorrinolaringólogos y/o intensivistas $(3,9)$.

En el algoritmo 3 de las guías se revisa el manejo en el caso de no ventilaciónno intubación, el cual es similar a la población no grávida, excepto que las embarazadas requieren soporte vital avanzado específico y cesárea perimortem, si la cricotiroidotomía fracasa (Maeda, 3).

Previo a la preparación de abordaje cervical quirúrgico de emergencia, debe descartarse el laringoespasmo (9), es preciso continuar administrando $\mathrm{O} 2$ al $100 \%$, garantizar el bloqueo neuromuscular adecuado y mantener la posición de la paciente con el cuello extendido (11). En este sentido, es primordial tomar en cuenta que la membrana cricotiroidea es profunda, en especial en pacientes con obesidad mórbida, por lo que puede ser complicado palparla e identificarla. En ese caso, la ultrasonografía en el punto de atención (POCUS) es la mejor estrategia para identificar la membrana $(1,3)$. Una vez que se realiza el procedimiento, se requiere evaluar si la oxigenación se restableció: si no ocurrió, se debe brindar soporte vital materno avanzado o practicar una cesárea perimortem, según corresponda. Si se logró la oxigenación, se debe decidir si es seguro y necesario proceder con la cirugía en ese momento: si resulta necesario y seguro, se efectúa con el procedimiento; si no lo es, se debe despertar a la paciente $(1,3)$.

\section{Extubación}

Alrededor del $30 \%$ de eventos adversos que ocurren durante una anestesia 
suceden al final de esta o durante la fase de recuperación.

Estos pueden ser: aspiración pulmonar, obstrucción de la vía aérea o hipoventilación $(3,9)$. Es por esta razón que en los algoritmos establecidos en las guías un punto esencial es planificar cómo ocurrirá la extubación desde el momento en que se comprueba que la intubación fue exitosa (9). La extubación en obstetricia debe realizarse con la paciente despierta, capaz de obedecer órdenes y ventilando un volumen corriente adecuado. Clásicamente, las pacientes para la extubación eran posicionadas en decúbito lateral izquierdo y Trendelemburg por su riesgo de aspiración, sin embargo, más recientemente, se ha demostrado que la posición de Fowler mejora la permeabilidad de la vía aérea, la función respiratoria y provee un mejor acceso a la vía aérea, especialmente en pacientes obesas. De igual modo, como se ha establecido que un tercio de las aspiraciones ocurren durante la extubación, se sugiere realizar previamente aspiración gástrica mediante sondaje (17).

\section{EQUIPO}

Como parte del equipo que debe estar disponible de rutina para manejo de la vía aérea destaca lo siguiente: una fuente de oxígeno, máscara con bolsa y válvula de no reinhalación, laringoscopios, tubos endotraqueales con guías, otros dispositivos nasales, orales y supraglóticos, equipo de succión, oxímetro de pulso, capnografía, estetoscopio, cinta adhesiva, monitores de presión arterial y electrocardiografía, acceso intravenoso y carro de paro $(10,18)$. Además de esto, se debe contar con un carro de vía aérea difícil, el cual debe contener:

- Equipo básico: máscaras faciales, cánulas orofaríngeas, 2 mangos de laringoscopio, hojas Macintosh tamaño 3 y 4 , tubos endotraqueales $6.5-7.0 \mathrm{~mm}$ con estilete, jeringas del $10 \mathrm{~mL}$ vacías para inflar el balón, tubos endotraqueales de reserva de diferentes tamaños, dispositivos supraglóticos de segunda generación de diferentes tamaños y tipos, junto con un dispositivo de succión $(1,6)$.

- Equipo secundario: otros tipos de laringoscopios (hojas McCoy, Miller), videolaringoscopios, estiletes ópticos, broncoscopio fibróptico, equipo de cricotiroidotomía $(1,6)$.

- Drogas para intubación: propofol, midazolam, etomidato, ketamina, rocuronio, sugammadex y succinilcolina, aunque empleando esta última es muy probable que la hipoxia ocurra antes de que desaparezcan sus efectos en un caso de no ventilación-no intubación, además de que sus propiedades despolarizantes también aumentan el consumo de oxígeno $(1,9)$.

\section{CONCLUSIONES}

A través de la elaboración de este artículo, se evidenció que la anatomía y fisiología respiratoria de las mujeres embarazadas varían considerablemente con respecto a la población no grávida, principalmente, como consecuencia de edema de la vía aérea, cambios en los volúmenes y capacidades pulmonares, aumento del volumen intratorácico, desplazamiento de estructuras abdominales, entre otras alteraciones, las 
cuales, en conjunto, acrecientan el riesgo de broncoaspiración, obstaculizan la visualización laríngea, agravan el riesgo de trauma y facilitan la desaturación durante episodios de apnea, por lo que las pacientes obstétricas deben ser consideradas vía aérea difícil.

Debido a lo anterior, en estas pacientes es fundamental llevar a cabo una evaluación rutinaria completa previo a la manipulación de la vía aérea, en busca de predictores de difícil ventilación o intubación, los cuales coinciden con los factores evaluados en otros grupos de población, como los siguientes: obesidad, Mallampati, grosor y longitud del cuello, tamaño de los incisivos superiores, distancia tiromentoniana, entre otros.

Con respecto al manejo de la vía aérea en las pacientes obstétricas, es forzoso tomar ciertas precauciones adicionales, por ejemplo: seleccionar tubos endotraqueales más pequeños, evitar la ruta nasal, optimizar el posicionamiento de la paciente, enfatizar la preoxigenación, preferir la secuencia de intubación rápida y considerar dispositivos avanzados.

De igual modo, se han elaborado guías donde se plantean algoritmos que orientan el manejo de la vía aérea en este tipo de pacientes, en los cuales se subraya la trascendencia de la planificación y preparación, asimismo, se describen las mejores prácticas para la secuencia de intubación rápida y la laringoscopía, además de detallar los procedimientos en caso de que la intubación y/o la ventilación fallen, limitando las oportunidades de intubación de 2 a 3 intentos únicamente antes de cambiar de estrategia. Igualmente, planear la extubación es otro procedimiento esencial que se destaca en la bibliografía, debido al riesgo de aspiración que conlleva.

Finalmente, es preciso recordar que, pese a que existen protocolos que enumeran pasos para el abordaje de la vía aérea en embarazadas, las acciones que se tomen van a depender en gran medida de las condiciones maternas y fetales, que son las que deben orientar el proceder del médico.

\section{REFERENCIAS}

1. Einav S, Weiniger CF, Landau R, editors. Principles and Practice of Maternal Critical Care. Switzerland: Springer; 2020.

2. Mushambi MC, Athanassoglou V, Kinsella SM. Anticipated difficult airway during obstetric general anaesthesia: narrative literature review and management recommendations. Anaesthesia. 2020; 75: 945-961. https://doi.org/10.1111/anae.15007

3. Mushambi MC, Kinsella SM, Popat M, Swales H, Ramaswamy KK, Winton AL, et al. Obstetric Anaesthetists' Association and Difficult Airway Society guidelines for the management of difficult and failed tracheal intubation in obstetrics. Anaesthesia. 2015 Nov; 70(11):1286-306. https://doi.org/10.1111/anae.13260.

4. Ramírez C, Rivera R, Tovar L. Claves para el manejo de la vía aérea en la embarazada. Rev Mex Anest. 2016; 39(1): 64-70.

5. Singhal V. Chapter 40: Pregnancy. In: Prabhakar H, editor. Essentials of Neuroanesthesia. London: Elsevier; 2017. p.669-680.

6. Alanoğlu Z, Erkoç SK, Güçlü ÇY, Meço BC, Baytaş V, Can ÖS, et al. Challenges of obstetric anesthesia: difficult laryngeal visualization. Acta Clin Croat. 2016 Mar; 55(1): 68-72. https://doi.org/10.20471/acc.2016.55.s1.09 
7. Bennet TA, Katz VL, Zelop CM. Cardiac Arrest and Resuscitation Unique to Pregnancy. Obstet Gynecol Clin N Am. 2016 Dec; 43(4): 809-819. http://dx.doi.org/10.1016/j.ogc.2016.07.011

8. Crozier TME. General Care of the Pregnant Patient in the Intensive Care Unit. Semin Respir Crit Care Med. 2017; 38: 208-217. https://doi.org/10.1055/s-0037-1600905

9. Roshan F, Phillips S. El Manejo de la Intubación traqueal difícil y/o fallida en anestesia obstétrica. Rev Chil Anest. 2016; 45(1): 16-26. https://doi.org/10.25237/revchilanestv45n01.02

10. Butterworth JF, Mackey DC, Wasnik JD. Morgan \& Mikhail's Clinical Anesthesiology. United States: McGraw-Hill; 2018.

11. Lambertus TE, Gómez B, Ottenwalder M. Protocolo de atención para el manejo de la vía aérea difícil [Internet]. República Dominicana: Ministerio de Salud Pública; 2017 [citado 2020 Dic 28]. Disponible en: https://repositorio.msp.gob.do/handle/123456789/932

12. Lapinsky S. Management of Acute Respiratory Failure in Pregnancy. Semin Respir Crit Care Med. 2017; 38: 201207. https://doi.org/10.1055/s-0037-1600909

13. Maeda A, Fujita N, Nagasaka Y. Respiratory and Airway Considerations in Obstetric Patients. Current Anesthesiology Reports. 2019; 9: 48-54. https://doi.org/10.1007/s40140-019-00309-0

14. McGuire B, Lucas DN. Planning the obstetric airway. Anaesthesia. 2020; 75(7): 852-855. https://doi.org/10.1111/anae.14987

15. Metodiev Y, Mushambi M. Supraglottic airway devices for Caesarean delivery under general anaesthesia: for all, for none, or for some?. Br J Anaesth. 2020; 125(1): e7-e11. https://doi.org/10.1016/j.bja.2020.02.012

16. Ramkumar V, Dinesh E, Shetty SR, Kundra P, Das S, Myatra SM, et al. All India Difficult Airway Association 2016 guidelines for the management of unanticipated difficult tracheal intubation in obstetrics. Indian J Anaesth 2016; 60:899-905. https://doi.org/10.4103/0019-5049.195482

17. Rojas A, Balkenhol M, Herrera O, Opazo M, Hernández M, Rivera M. Síndrome de Hallermann-Streiff y embarazo: manejo de la vía aérea difícil en embarazadas. Rev Chil Obstet Ginecol. 2016; 81(3): 223-228. http://dx.doi.org/10.4067/S0717-75262016000300009

18. Alvarado M. Secuencia de intubación rápida en el Servicio de Emergencias: una revisión bibliográfica. Revista de la Facultad de Medicina de la Universidad de Iberoamérica. 2020; 3(2): 1-9. 